IJEMD-M, I (1) (2022) $17-37 \quad \underline{\text { https://doi.org/ } 10.54938 / \text { ijemdm.2022.01.1.7 }}$ International Journal of Emerging Multidiciplinaries
Mathematics
Research Paper
Journal Homepage: $: \underline{w w . i j e m d . c o m}$
ISSN (print): $2790-1998$

\title{
Novel Techniques for Solving Goursat Partial Differential Equations in the Linear and Nonlinear Regime
}

\author{
Amina Iftikhar ${ }^{1}$, Wajeeha Arif ${ }^{1}$, Laiba Zaman', and Tahir Naseem ${ }^{1, *}$ \\ ${ }^{I}$ Department of Mathematics, Government Postgraduate College Haripur, 22620, Pakistan \\ *Corresponding author
}

\begin{abstract}
The Goursat problem, which is related to hyperbolic partial differential equations, occurs in a variety of branches of physics and engineering. We studied the solution of the Goursat partial differential equation utilizing the reduced differential transform (RDT) and Adomian decomposition (AD) techniques in this inquiry. The problem's analytical solution is found in series form, which converges to exact solutions. The approaches' reliability and efficiency were evaluated using the Goursat problems (linear and non-linear). Additionally, the accuracy of the findings obtained demonstrates the reduced differential approach's superiority over the Adomian decomposition method and other numerical methods previously applied to the Goursat problem.
\end{abstract}

Keywords: Partial Differential equation; series solution; Goursat problem; RD method; AD method.

\section{Introduction}

French mathematician Goursat [1] coined the name for a linear problem of the kind $u_{x t}=a(x, t) u_{x}+$ $b(x, t) u_{t}+c(x, t) u_{t}+c(x, t) u+f(x, t)$. The Goursat problem, which is related to hyperbolic partial differential equations, occurs in a variety of branches of physics and engineering. Cannon [2] was the first to draw attention to these problems with integral circumstances, and Samarskii [3] emphasized the relevance of the problem with integral conditions. Numerous numerical approaches have been used to study the 
Goursat equation, including the finite difference method [4], the Runge-Kutta method [5], and the Homotopy Analysis Method (HAM) [6]. The differential transform (DT) method is a technique for addressing a wide variety of problems whose mathematical models yield algebraic, differential, integral, or integro-differential equations or systems of equations [7-10]. There are numerous uses of Zhou [11]'s differential transform in electric circuit analysis, including solving both linear and nonlinear initial value problems. This method generates a polynomial-based analytical answer. The differential transform method (DTM) is an iterative procedure for obtaining Taylor series solutions to differential equations. As a result of this procedure, an analytical solution in the form of polynomials is constructed.

Gorge Adomian's [12-14] introduced a strategy for solving partial differential equations of any type (algebraic, differential, integro-differential, and integral) as well as stochastic situations. Since then, this procedure has been referred to as Adomian's Decomposition Method (ADM). As an initial approximation, identify the initial or boundary data and terms involving the independent variable alone, decomposing the non-linear function in terms of Adomian's polynomial, and finding the successive terms of the series solution via recurrence relations. Numerous studies [15-18] examined convergence and also resulted in modifications to the Adomian decomposition approach that were later applied to various forms of ordinary and partial differential equations by subsequent scientists.

The first section of this paper has a brief introduction; the second section contains an analysis of the RDTM; the third section contains an analysis of the ADM; the fourth section contains examples of Goursat problems handled using both approaches; and the final section contains a conclusion.

\section{2. $\quad$ Reduction Differential Transform (RDT) Analysis}

The following are the fundamental definitions of the reduced differential transform method.

2.1. Definition [19] Assume, for example, that the function $u(x, t)$ is analytic and continuously differentiable in time $\mathrm{t}$ and space $x$.

$$
U_{k}(x)=\frac{1}{k !}\left[\frac{d^{k} u(x, t)}{d t^{k}}\right]_{t=0},
$$

$t$-dimensional spectrum functions are found. The converted function is $U_{k}(x)$. According to this definition, the differential inverse of $U_{k}(x)$ is

$$
u(x, t)=\sum_{k=0}^{\infty} U_{k}(x) t^{k}
$$


If we combine Equations (2.1) and (2.2), we get

$$
u(x, t)=\sum_{k=0}^{\infty} \frac{1}{k !}\left[\frac{d^{k} u(x, t)}{d t^{k}}\right]_{t=0} t^{k} .
$$

The following table lists the functions and their reduced differential transformations.

Table 1. Functions and the modifications that they undergo.

\begin{tabular}{|c|c|c|c|}
\hline Function & Transformation & Function & Transformation \\
\hline$u(x, t)$ & $U_{k}(x)=\frac{1}{k !}\left[\frac{d^{k} u(x, t)}{d t^{k}}\right]$ & $u(x, t) v(x, t)$ & $\sum_{r=0}^{k} U_{r}(x) V_{k-r}(x)$ \\
\hline$u(x, t) \pm v(x, t)$ & $U_{k}(x) \pm V_{k}(x)$ & $\frac{d^{r} u(x, t)}{d t^{r}}$ & $\frac{(k+1) !}{k !} U_{k+1}(x)$ \\
\hline$\propto u(x, t)$ & $\propto U_{k}(x)(\propto i s$ constant $)$ & $\frac{d u(x, t)}{d x}$ & $\frac{d U_{k}(x)}{d x}$ \\
\hline$x^{m} t^{n}$ & $x^{m} \delta(k-n)$ & $\frac{d^{r+s} u(x, t)}{d x^{r} d t^{s}}$ & $(k+s) \frac{d U_{k+s}(x)}{d x^{r}}$ \\
\hline$x^{m} t^{n} u(x, t)$ & $x^{m} U(k-n)$ & & \\
\hline
\end{tabular}

\section{An analysis of Adomian's Decomposition (AD) Method [20]}

We will consider the following nonlinear differential equation in order to demonstrate the solution procedure of the Adomian's Decomposition method (ADM).

$$
L_{x} L_{t} u(x, t)=f(x, t)+u^{n}(x, t),
$$

with the condition

$$
\left.\begin{array}{c}
u(x, 0)=g(x), \\
u(0, t)=h(t), \\
u(0,0)=a
\end{array}\right\},
$$

where $L_{x}=\frac{\partial}{\partial x}, L_{y}=\frac{\partial}{\partial t}$, with the inverse operator $L_{x}^{-1} L_{t}^{-1}()=.\int_{0}^{x} \int_{0}^{t}() d x d$.$t .$ 
The solution to the problem is supposed to be as follows:

$$
u(x, t)=\sum_{n=0}^{\infty} u_{n}(x, t)
$$

The nonlinear function is considered as

$$
u^{n}(x, t)=\sum_{n=0}^{\infty} A_{n}(x, t)
$$

where $A_{n}(x, t)$, are referred to as Adomian's polynomials, and they are defined using the following scheme.

$$
\begin{gathered}
A_{0}=\mathrm{F}\left(\mathrm{u}_{0}\right), \\
A_{1}=u_{1} F^{\prime\left(u_{0}\right)}, \\
A_{2}=u_{2} F^{\prime}\left(u_{0}\right)+\frac{u_{1}^{2}}{2 !} F^{\prime \prime}\left(u_{0}\right) \\
\cdot \\
A_{n}=\frac{1}{n !} \frac{d^{n}}{d \lambda^{n}} N\left(\sum_{i=0}^{\infty} \lambda^{i} u_{i}\right)_{\lambda=0}, n=0,1,2,3, . .
\end{gathered}
$$

The components of $u_{n}(x, t)$ are completely determined, by the recurrent relationship

$$
\begin{gathered}
u_{0}=g(x)+h(x)-a+f(x), \\
u_{n}=\int_{0}^{x} \int_{0}^{t} A_{n-1} d t d x, \quad n \geq 1 .
\end{gathered}
$$

As a result, the exact answer can be derived by employing Eq (6).

\section{Implementation of Reduced Differential Transform Method}

We implemented RDT and AD methods on linear and non-linear Goursat problems which are given as under:

Example 4.1 Consider the homogenous Goursat problem

$$
u_{x t}=u,
$$

with the given conditions 


$$
u(x, 0)=e^{x}, u(0, t)=e^{t}, u(0,0)=1 .
$$

and the problem has the exact solution

$$
u(x, t)=e^{x+t}
$$

Now, by using reduced differential transform method from table 2, the equation (12) and (13) takes the form

$$
\begin{gathered}
U_{0}=e^{x}, \\
(k+1) \frac{d U_{k+1}(x)}{d x}=U_{k}, \text { for } \mathrm{k} \geq 0,
\end{gathered}
$$

By applying the above procedure we obtain

$$
U_{1}=e^{x}, U_{1}=e^{x}, U_{3}=\frac{e^{x}}{3 !}, \ldots
$$

Finally by applying the inverse reduced transforms we have

$$
\begin{gathered}
u(x, t)=\sum_{k=0}^{\infty} U_{k}(x) t^{k}, \\
u(x, t)=\sum_{k=0}^{\infty} \frac{e^{x}}{k !} t^{k} \\
u(x, t)=e^{x}+\frac{e^{x}}{1 !} t+\frac{e^{x}}{2 !} t^{2}+\frac{e^{x}}{3 !} t^{3}+\cdots \\
u(x, t)=e^{x+t} .
\end{gathered}
$$

The graphs of RDTM with $10^{\text {th }}$ iterations and of exact solution are given as:

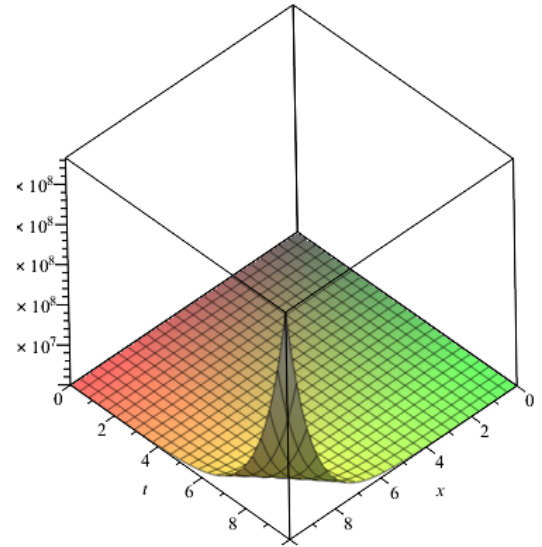

Fig. 1(a). Graphical presentation of $10^{\text {th }}$ iteration of RDTM.

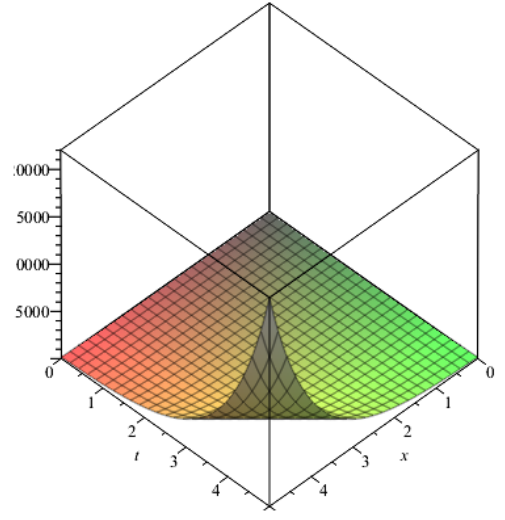

Fig. 1(b). Graphical presentation of exact solution. 
Example 4.2 Consider the homogenous Goursat problem

$$
u_{x t}=-2 u
$$

with the given conditions

$$
u(x, 0)=e^{x}, u(0, t)=e^{t}, u(0,0)=1
$$

and the problem has the exact solution

$$
u(x, t)=e^{x-2 t}
$$

Now, by using reduced differential transform method from table 2, the equation (18) and (19) takes the form

$$
\begin{gathered}
U_{0}=e^{x}, \\
(k+1) \frac{d U_{k+1}(x)}{d x}=-2 U_{k},
\end{gathered}
$$

By applying the above procedure we obtain

$$
U_{0}=e^{x}, U_{1}=-2 e^{x}, U_{2}=2 e^{x}, \ldots
$$

Finally by applying the inverse reduced transforms we have

$$
\begin{gathered}
u(x, t)=\sum_{k=0}^{\infty} U_{k}(x) t^{k} \\
u(x, t)=\left[e^{x}+\left(-2 e^{x}\right)+\left(2 e^{x}\right)+\left(-\frac{4}{3} e^{x}\right)+\left(\frac{2}{3} e^{x}\right)+\left(-\frac{4}{15} e^{x}\right)+\cdots\right] t^{k}, \\
u(x, t)=e^{x-2 t} .
\end{gathered}
$$

The graphs of RDTM with $10^{\text {th }}$ iterations and of exact solution are given as

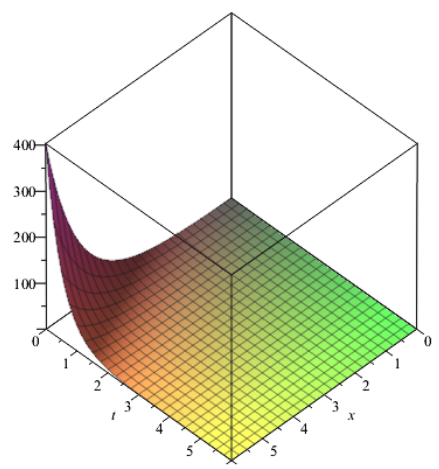

Fig. 2(a). Graphical presentation of $10^{\text {th }}$ iteration of RDTM.

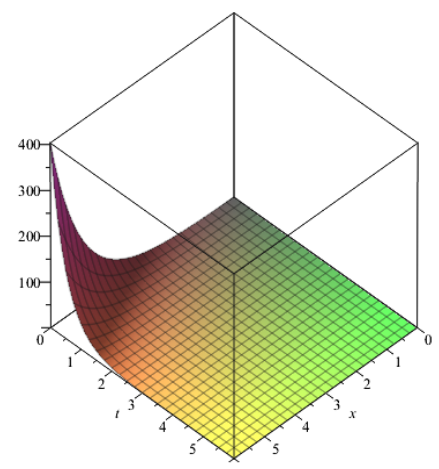

Fig. 2(b). Graphical presentation of exact solution. 
Example 4.3 The linear inhomogeneous Goursat problem

$$
u_{x t}=u-t
$$

with the conditions

$$
u(x, 0)=e^{x}, u(0, t)=t+e^{t}, u(0,0)=1
$$

Equations (24) and (25) assume the form when the reduced differential transform method from table 2 is used.

$$
\begin{gathered}
U_{0}=t+e^{t} \\
(k+1) \frac{d U_{k+1}(x)}{d x}=U_{k}-t \delta(k),
\end{gathered}
$$

By applying the above procedure we obtain

$$
U_{0}=t+e^{t}, U_{1}=e^{t}, U_{2}=\frac{e^{t}}{2}, \ldots
$$

Finally by applying the inverse reduced transforms we have

$$
\begin{gathered}
u(x, t)=\sum_{k=0}^{\infty} U_{k}(x) t^{k}, \\
u(x, t)=\left[\left(t+e^{t}\right) x^{0}+e^{t} x+\frac{e^{t}}{2 !} x^{2}+\frac{e^{t}}{3 !} x^{3}+\frac{e^{t}}{4 !} x^{4}+\cdots\right], \\
u(x, t)=t+e^{t+x} .
\end{gathered}
$$

The graphs of RDTM with $10^{\text {th }}$ iterations and of exact solution are given as

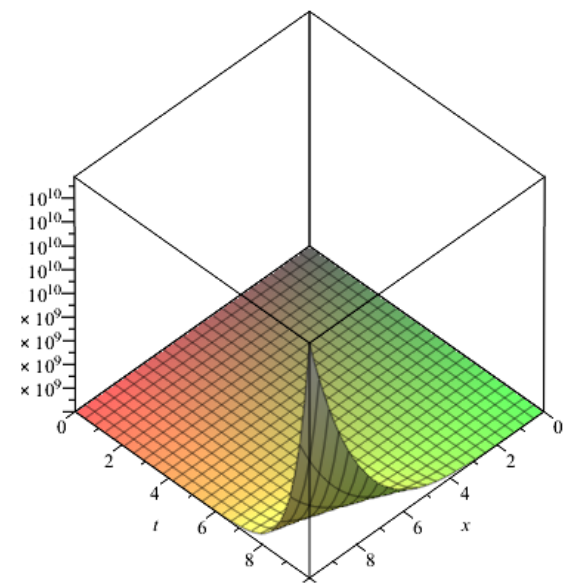

Fig. 3(a). Graphical presentation of $10^{\text {th }}$ iteration of RDTM.

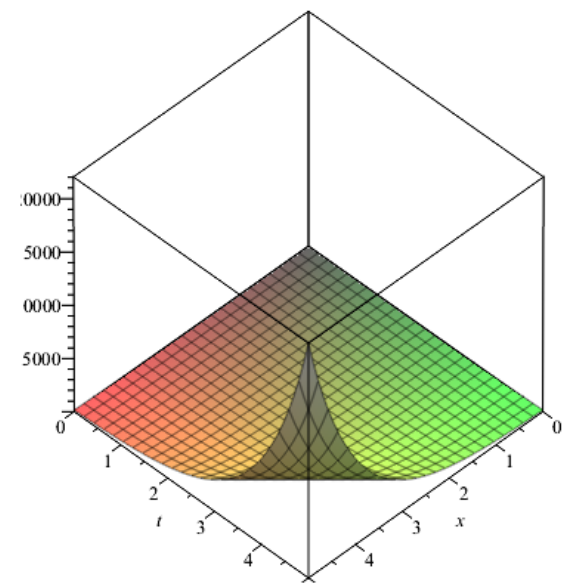

Fig. 3(b). Graphical presentation of exact solution. 
Example 4.4 Consider the inhomogeneous linear Goursat equation

$$
u_{x t}=u+4 x t-x^{2} t^{2}
$$

subject to the conditions

$$
u(x, 0)=e^{x}, u(0, t)=e^{t}, u(0,0)=1,
$$

By using the reduced differential transform method described in table 2, equations (30) and (31) now take the form

$$
\begin{gathered}
U_{0}=e^{x} \\
(k+1) \frac{d U_{k+1}(x)}{d x}=U_{k}+4 x \delta(k-1)-x^{2} \delta(k-2),
\end{gathered}
$$

By applying the above procedure we obtain

$$
U_{0}=e^{x}, U_{1}=e^{x}, U_{2}=\frac{e^{x}}{2}+x^{2}, \ldots
$$

Finally by applying the inverse reduced transforms we have

$$
\begin{gathered}
u(x, t)=\sum_{k=0}^{\infty} U_{k}(x) t^{k} \\
u(x, t)=x^{2} t^{2}+\left[e^{x}\left(1+t+\frac{t^{2}}{2 !}+\frac{t^{3}}{3 !}+\right)\right], \\
u(x, t)=x^{2} t^{2}+e^{x+t}
\end{gathered}
$$

The graphs of RDTM with $10^{\text {th }}$ iterations and of exact solution are given as

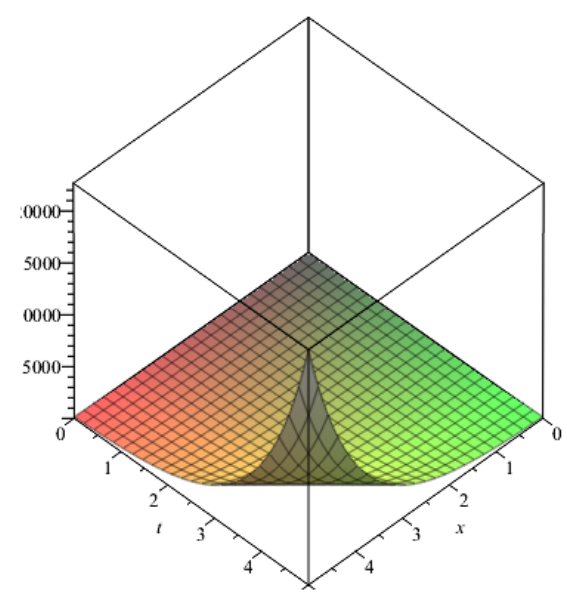

Fig. 4(a). Graphical presentation of $10^{\text {th }}$ iteration of RDTM.

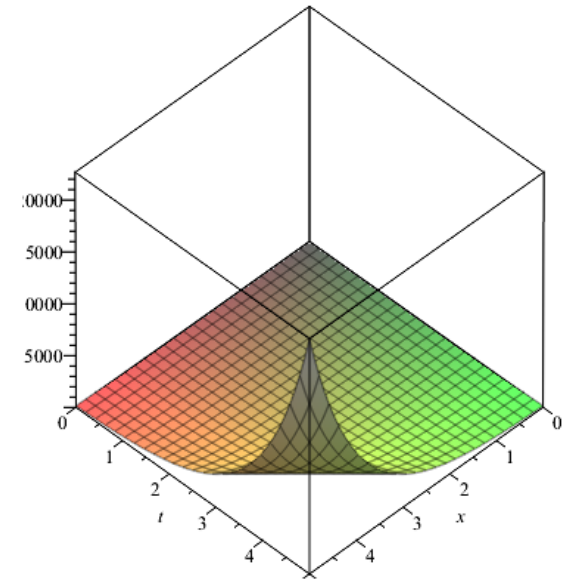

Fig. 4(b). Graphical presentation of exact solution. 
Example 4.5 The non-linear Goursat problem

$$
u_{x t}=-u^{3}+x^{3}+3 x^{2} t+3 x t^{2}+t^{3}
$$

with the conditions

$$
u(x, 0)=x, u(0, t)=t, u(0,0)=0 .
$$

Equations (36) and (37) assume the form when the reduced differential transform method from Table 2 is used

$$
\begin{gathered}
U_{0}=x, \\
U_{r}=\sum_{s=0}^{r} U_{r} U_{r-s}, \\
(k+1) \frac{d U_{k+1}}{d x}=-\sum_{r=0}^{k} u_{r}^{2} u_{k-r}+x^{3} \delta(k)+3 x^{2} \delta(k-1)+3 x \delta(k-2)+\delta(k-3)
\end{gathered}
$$

By applying the above procedure we obtain

$$
U_{1}=1, U_{2}=U_{3}=U_{4}=U_{5}=0=\cdots
$$

Finally by applying the inverse reduced transforms we have

$$
\begin{gathered}
u(x, t)=\sum_{k=0}^{\infty} U_{k}(x) t^{k}, \\
u(x, t)=[x+1+0+\cdots] t^{k}, \\
u(x, t)=x+t .
\end{gathered}
$$

The graphs of RDTM with $10^{\text {th }}$ iterations and of exact solution are given as 


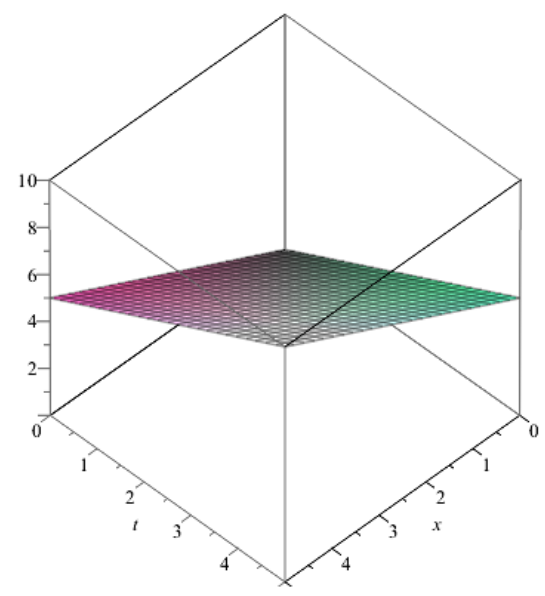

Fig. 5(a). Graphical presentation of $10^{\text {th }}$ iteration of RDTM.

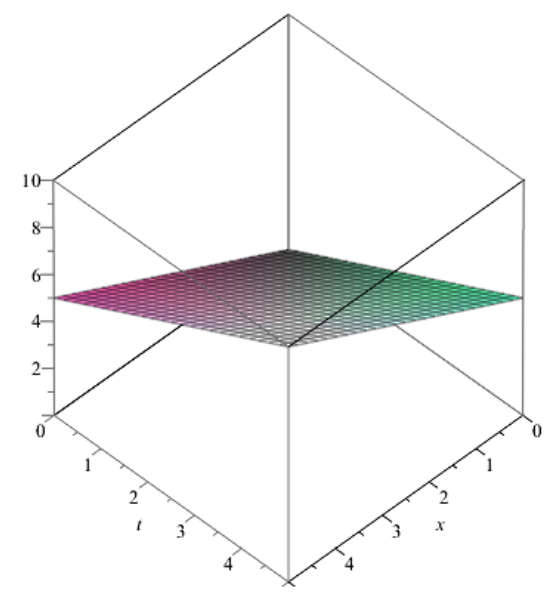

Fig. 5(b). Graphical presentation of exact solution.

Example 4.6 Let us suppose the non-linear Goursat problem

$$
u_{x t}=e^{-2 u}
$$

Subject to the conditions

$$
u(x, 0)=0, u(0, t)=0, u(0,0)=0 .
$$

Equations (43) and (44) assume the form when the reduced differential transform method from table 2 is used

$$
\begin{gathered}
U_{0}=0, \\
(k+1) \frac{d}{d x} U_{k+1}=N_{k}\left(U_{k}\right),
\end{gathered}
$$

where $N\left(U_{k}\right)$ is the obtained by using Adomian polynomial for $e^{-2 u}$, such that

$$
\begin{gathered}
N_{0}=f\left(U_{0}\right)=e^{-2 U_{0}}, \\
N_{1=} f^{\prime}\left(U_{0}\right) U_{1}=-2 e^{-2 U_{0}} U_{1}, \\
N_{2}=f^{\prime}\left(U_{0}\right) U_{2}+f^{\prime \prime}\left(U_{0}\right) \frac{U_{1}^{2}}{2 !}=-2 e^{-2 U_{0}} U_{2}+4 e^{-2 U_{0}} \frac{U_{1}^{2}}{2 !},
\end{gathered}
$$

and so on

By applying the above procedure we obtain

$$
U_{1}=x, U_{2}=-\frac{x^{2}}{2}, U_{3}=\frac{x^{3}}{3}, . .
$$

Finally by applying the inverse reduced transforms we have 


$$
\begin{gathered}
u(x, t)=\sum_{k=0}^{\infty} U_{k}(x) t^{k} \\
u(x, t)=\left[x t-\frac{x^{2} t^{2}}{2}+\frac{x^{3} t^{3}}{3}-\frac{x^{4} t^{4}}{4}+\cdots\right] \\
u(x, t)=\ln (1+x t) .
\end{gathered}
$$

The graphs of RDTM with $10^{\text {th }}$ iterations and of exact solution are given as

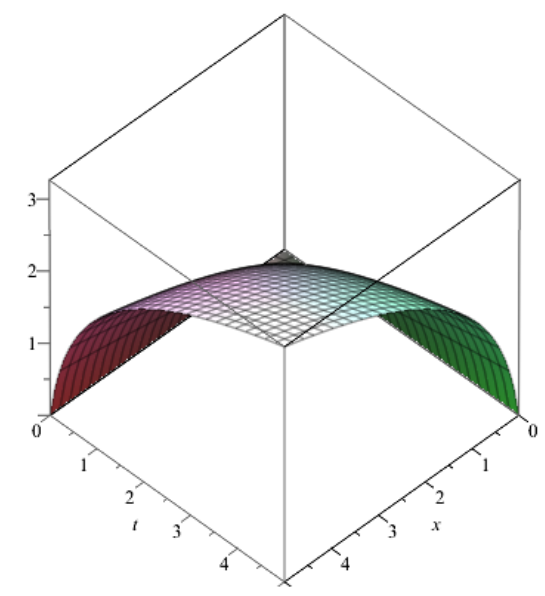

Fig. 6(a). Graphical presentation of $10^{\text {th }}$ iteration of RDTM.

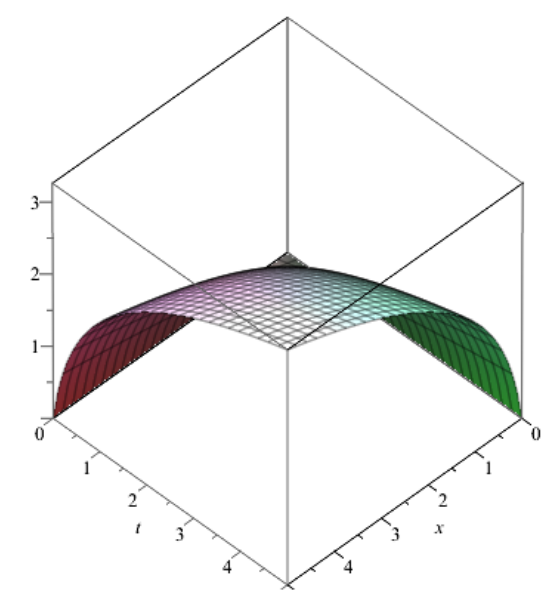

Fig. 6(b). Graphical presentation of exact solution.

\section{Implementation of Adomian's Decomposition Method}

In this section, we will use Adomian's decomposition (AD) method to solve linear and nonlinear Goursat problems.

Example 5.1 Let us suppose the following linear homogeneous Goursat Problem

$$
u_{x t}=u
$$

With the given conditions

$$
u(x, 0)=e^{x}, u(0, t)=e^{t}, u(0,0)=1 .
$$

and the problem has the exact solution

$$
u(x, t)=e^{x+t} .
$$

By using AD method, we get 


$$
\begin{gathered}
u(x, t)-u(0, t)-u(x, 0)+u(0,0)=\int_{0}^{x} \int_{0}^{t} u(x, t) d t d x \\
u(x, t)=e^{x}+e^{t}-1+\int_{0}^{x} \int_{0}^{t} u(x, t) d t d x .
\end{gathered}
$$

Consequently, we have

$$
u_{0}(x, t)=e^{x}+e^{t}-1, u_{1}(x, t)=x e^{t}+t e^{x}-x t-x-t, . .
$$

Hence by using equation (6), we have

$$
\begin{gathered}
u(x, t)=\sum_{k=0}^{\infty} u_{n}(x), \\
u(x, t)=e^{x}\left(1+t+\frac{t^{2}}{2 !}+\cdots\right)+e^{t}\left(1+x+\frac{x^{2}}{2 !}+\cdots\right)-\left(1+(x+t)+\frac{(x+t)^{2}}{2 !}+\cdots\right) \\
u(x, t)=e^{x+t} .
\end{gathered}
$$

The graphs of $\mathrm{ADM}$ with $10^{\text {th }}$ iterations and of exact solution are given as:

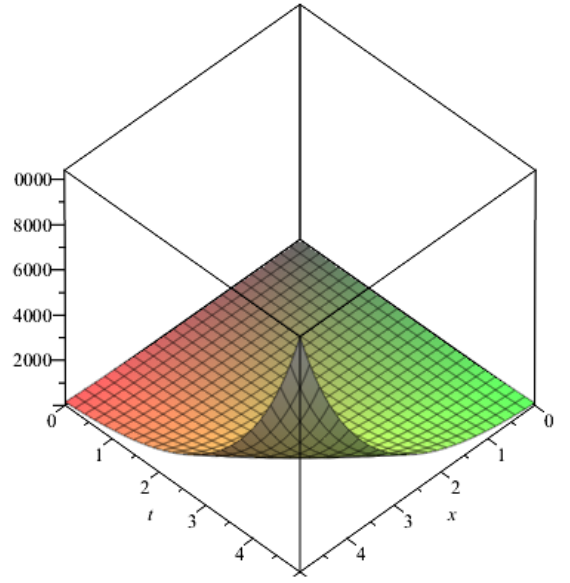

Fig. 7(a). Graphical presentation of $10^{\text {th }}$ iteration of ADM.

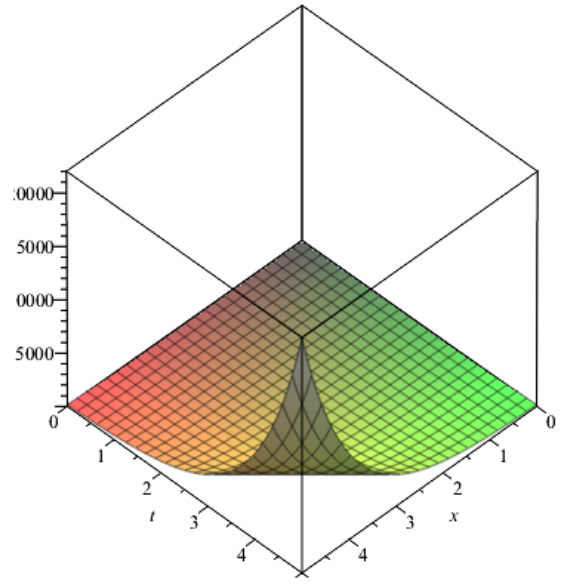

Fig. 7(b). Graphical presentation of exact solution.

Example 5.2 Suppose the following linear homogeneous Goursat Problem

$$
u_{x t}=-2 u
$$

with the given conditions 


$$
u(x, 0)=e^{x}, u(0, t)=e^{t}, u(0,0)=1
$$

and the problem has the exact solution

$$
u(x, t)=e^{x-2 t}
$$

By using AD method, we get

$$
\begin{gathered}
u(x, t)-u(0, t)-u(x, 0)+u(0,0)=\int_{0}^{x} \int_{0}^{t} u(x, t) d t d x \\
u(x, t)=e^{x}+e^{t}-1+\int_{0}^{x} \int_{0}^{t} u(x, t) d t d x
\end{gathered}
$$

Consequently, we have

$$
u_{0}(x, t)=e^{x}+e^{t}-1, u_{1}(x, t)=-2\left(x e^{t}+t e^{x}-x t-x-t\right), . .
$$

Hence by using equation (6), we have

$$
\begin{gathered}
u(x, t)=\sum_{k=0}^{\infty} u_{n}(x), \\
u(x, t)=e^{x}+e^{t}-1-2 x e^{t}-2 t e^{x}+2 x t+2 x+2 t-x^{2}-t^{2} e^{x}+\frac{x^{2} t^{2}}{2}+x^{2} t+\frac{t^{3}}{3}+x^{2}+t^{2} \\
-\frac{x^{3}}{3} e^{t}-\frac{t^{3}}{3} e^{x}+\frac{x^{3}}{3 !} \frac{t^{3}}{3}+\frac{x^{3}}{3} \frac{t^{2}}{2}+\frac{t^{4}}{12}+\frac{x^{3}}{3} t+x \frac{t^{3}}{3}+\frac{x^{3}}{3}+\frac{t^{3}}{3}+\cdots \\
u(x, t)=e^{x-2 t} .
\end{gathered}
$$

The graphs of ADM with $10^{\text {th }}$ iterations and of exact solution are given as

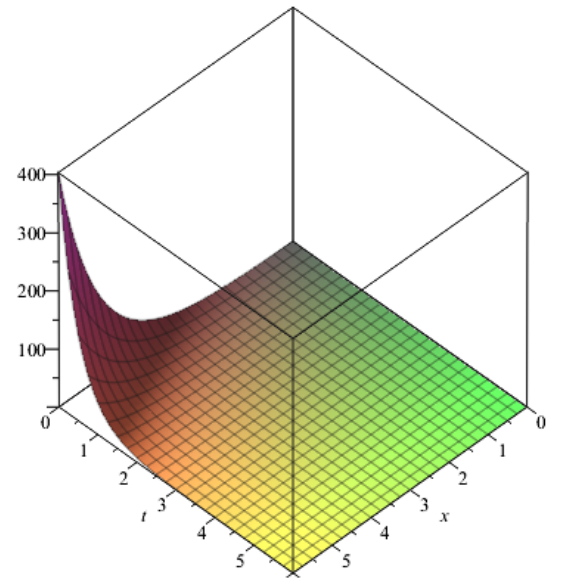

Fig. 8(a). Graphical presentation of $10^{\text {th }}$ iteration of ADM.

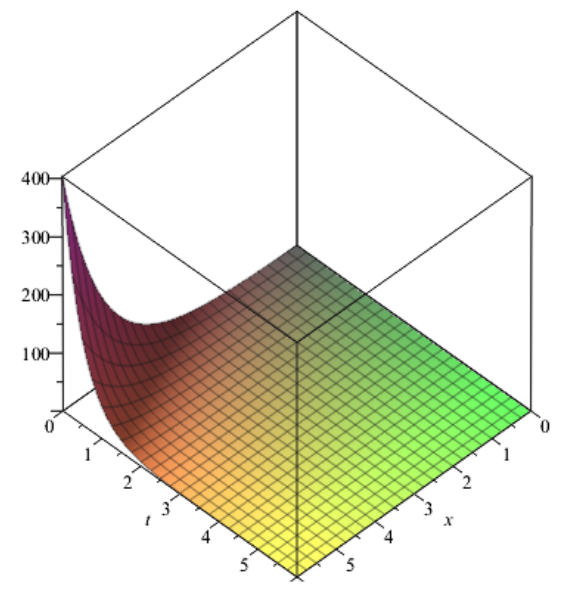

Fig. 8(b). Graphical presentation of exact solution. 
Example 5.3 Suppose the following linear inhomogeneous Goursat Problem

$$
u_{x t}=u-t
$$

subject to the conditions

$$
u(x, 0)=e^{x}, u(0, t)=t+e^{t}, u(0,0)=1
$$

and the exact solution is

$$
u(x, t)=t+e^{x+t}
$$

By using AD method, we get

$$
\begin{gathered}
u(x, t)=u(0, t)+u(x, 0)-u(0,0)-\frac{x t^{2}}{2}+\int_{0}^{x} \int_{0}^{t} u(x, t) d t d x, \\
u(x, t)=t+e^{t}+e^{x}-1-\frac{x t^{2}}{2}+\int_{0}^{x} \int_{0}^{t} u(x, t) d t d x .
\end{gathered}
$$

Consequently, we have

$$
u_{0}(x, t)=t+e^{t}+e^{x}-1-\frac{x t^{2}}{2}, u_{1}(x, t)=x \frac{t^{2}}{2}+x e^{t}+t e^{x}-x t-\frac{x^{2}}{2} \frac{t^{3}}{3 !}-x-t, .
$$

Hence by using equation (6), we have

$$
\begin{gathered}
u(x, t)=\sum_{k=0}^{\infty} u_{n}(x) \\
u(x, t)=t+e^{t}\left(1+x+\frac{x^{2}}{2 !}+\cdots\right)+e^{x}\left(1+t+\frac{t^{2}}{2 !}+\cdots\right)-\left(1+(x+t)+\frac{(x+t)^{2}}{2 !}+\cdots\right) \\
u(x, t)=t+e^{x+t} .
\end{gathered}
$$

The graphs of ADM with $10^{\text {th }}$ iterations and of exact solution are given as 


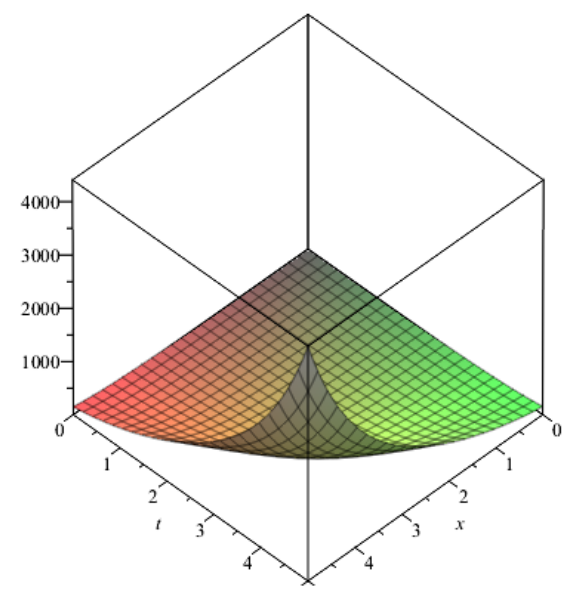

Fig. 9(a). Graphical presentation of $10^{\text {th }}$ iteration of ADM.

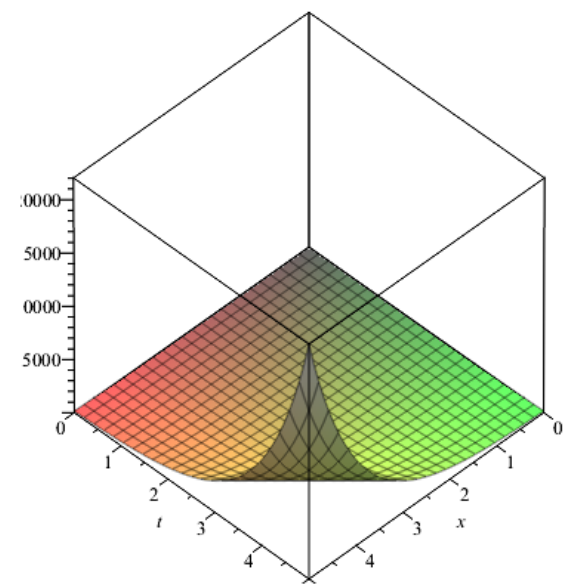

Fig. 9(b). Graphical presentation of exact solution.

Example 5.4 Suppose the following linear inhomogeneous Goursat Problem

$$
u_{x t}=u+4 x t-x^{2} t^{2}
$$

subject to the conditions

$$
u(x, 0)=e^{x}, u(0, t)=e^{t}, u(0,0)=1,
$$

and the exact solution is

$$
u(x, t)=x^{2} t^{2}+e^{x+t}
$$

By using $\mathrm{AD}$ method, we get

$$
\begin{gathered}
u(x, t)=u(0, t)+u(x, 0)-u(0,0)+4 \frac{x^{2}}{2} \frac{t^{2}}{2}-\frac{x^{3}}{3} \frac{t^{3}}{3}+\int_{0}^{x} \int_{0}^{t} u(x, t) d t d x, \\
u(x, t)=e^{x}+e^{t}-1+4 \frac{x^{2}}{2} \frac{t^{2}}{2}-\frac{x^{3}}{3} \frac{t^{3}}{3}+\int_{0}^{x} \int_{0}^{t} u(x, t) d t d x .
\end{gathered}
$$

Consequently, we have

$$
\begin{gathered}
u_{0}(x, t)=e^{x}+e^{t}-1+4 \frac{x^{2}}{2} \frac{t^{2}}{2}-\frac{x^{3}}{3} \frac{t^{3}}{3}, \\
u_{1}(x, t)=t e^{x}+x e^{t}-x t+\frac{x^{3} t^{3}}{9}-\frac{x^{4} t^{4}}{144}-x-t, . .
\end{gathered}
$$

Hence by using equation (6), we have 


$$
\begin{gathered}
u(x, t)=\sum_{k=0}^{\infty} u_{n}(x) \\
=x^{2} t^{2}+e^{x}\left[1+t+\frac{t^{2}}{2 !}+\cdots\right]+e^{t}\left[1+x+\frac{x^{2}}{2 !}+\cdots\right]-\left[1+(x+t)+\frac{(x+t)^{2}}{2 !}+\cdots\right] \\
u(x, t)=x^{2} t^{2}+e^{(x+t)} .
\end{gathered}
$$

The graphs of ADM with $10^{\text {th }}$ iterations and of exact solution are given as

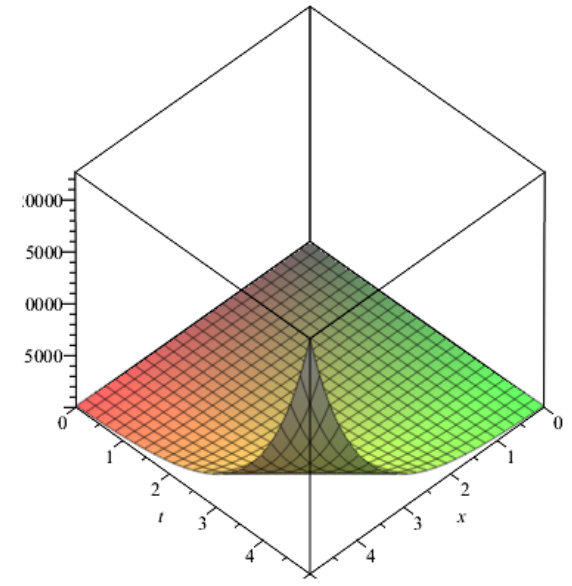

Fig. 10(a). Graphical presentation of $10^{\text {th }}$ iteration of ADM.

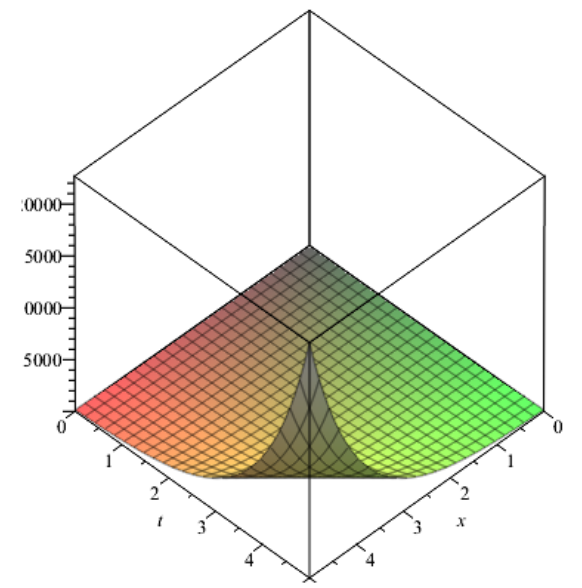

Fig. 10(b). Graphical presentation of exact solution.

Example 5.5 Suppose the following non-linear inhomogeneous Goursat problem

$$
u_{x t}=-u^{3}+x^{3}+3 x^{2} t+3 x t^{2}+t^{3}
$$

with the conditions

$$
u(0, t)=t, u(x, 0)=x, u(0,0)=0,
$$

and the problem has the exact solution

$$
u(x, t)=x+t .
$$

By using AD method, we get

$$
u(x, t)=x+t+\frac{x^{4} t}{4}+\frac{x^{3} t^{2}}{2}+\frac{x^{2} t^{3}}{2}+\frac{x t^{4}}{4}-\int_{0}^{x} \int_{0}^{t} A_{n} d t d x
$$

Consequently, we have 


$$
u_{0}(x, t)=x+t, u_{1}(x, t)=0, \ldots
$$

Hence by using equation (6), we have

$$
\begin{gathered}
u(x, t)=\sum_{k=0}^{\infty} u_{n}(x), \\
u(x, t)=x+t .
\end{gathered}
$$

The graphs of ADM with $10^{\text {th }}$ iterations and of exact solution are given as

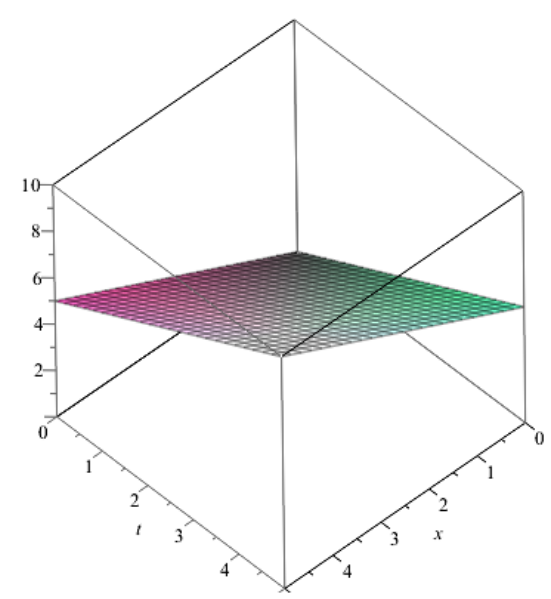

Fig. 11(a). Graphical presentation of $10^{\text {th }}$ iteration of ADM.

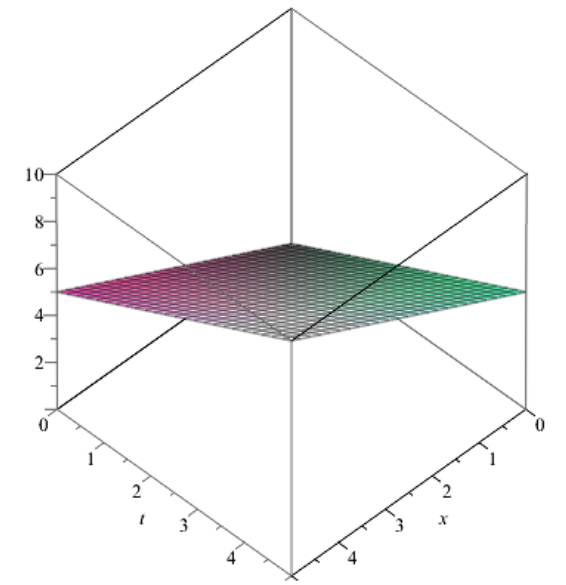

Fig. 11(b). Graphical presentation of exact solution.

Example 5.6: Suppose the non-linear Goursat problem

$$
u_{x t}=e^{-2 u}
$$

with the conditions

$$
u(x, 0)=0, u(0, t)=0, u(0,0)=0,
$$

and the problem has the exact solution

$$
u(x, t)=\ln (1+x t) .
$$

By using AD method, we get

$$
\begin{gathered}
u(x, t)=u(0, t)+u(x, 0)-u(0,0)+\int_{0}^{x} \int_{0}^{t} A_{n} d t d x, \\
u_{0}=0
\end{gathered}
$$


where $A_{n}$ is obtained by using polynomial for $e^{-2 u}$, such that

$$
\begin{gathered}
A_{0}=f\left(u_{0}\right)=e^{-2 u_{0}}, \\
A_{1=} f^{\prime}\left(u_{0}\right) u_{1}=-2 e^{-2 u_{0}}, \\
A_{3}=-2 e^{-2 u_{0}} u_{3}+4 e^{-2 u_{0}} u_{1} u_{2}-8 e^{-2 u_{0}} \frac{u_{1}^{3}}{3 !}, \ldots
\end{gathered}
$$

For $n=0$, equation $(81)$, becomes

$$
\begin{gathered}
u_{1}=\int_{0}^{x} \int_{0}^{t} A_{0} d t d x=x t, \\
u_{2}=\int_{0}^{x} \int_{0}^{t} A_{1} d t d x=-\frac{x^{2} t^{2}}{2}, \ldots . .
\end{gathered}
$$

Hence by using equation (6), we have

$$
\begin{gathered}
u(x, t)=\sum_{k=0}^{\infty} u_{n}(x), \\
u(x, t)=\left[x t-\frac{x^{2} t^{2}}{2}+\frac{x^{3} t^{3}}{3}+\cdots\right], \\
u(x, t)=\ln (1+x t) .
\end{gathered}
$$

The graphs of ADM with $10^{\text {th }}$ iterations and of exact solution are given as 


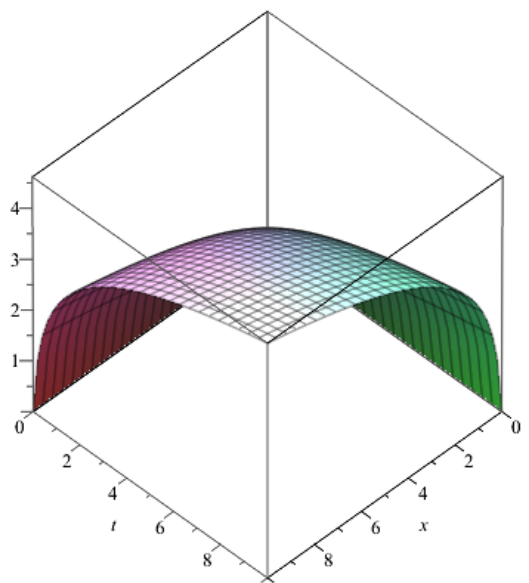

Fig. 12(a). Graphical presentation of $10^{\text {th }}$ iteration of ADM.

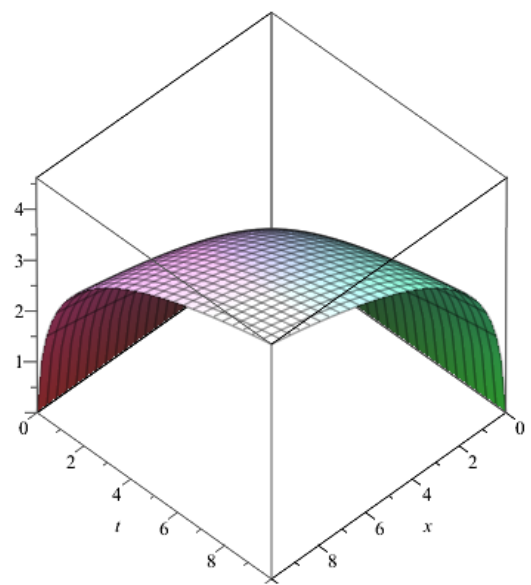

Fig. 12(b). Graphical presentation of exact solution.

\section{Conclusion}

Here in this investigation we use the reduced differential transform method and Adomian decomposition method for the solution of linear and nonlinear Goursat problem. We applied the both proposed methods on six Goursat problems. We compared the results of reduced differential transform and Adomian's decomposition methods with the exact solutions graphically. It is shown that the reduced differential transform method solves the linear and nonlinear Goursat problem without employing Adomian polynomials. Moreover, the reduced differential transform series solution converges faster than the Adomian decomposition solution. It is also found that reduced differential transform approach reduces the computational difficulties of Adomian decomposition method.

\section{References}

[1] Goursat, E. A Course in Mathematical Analysis: pt. 1. Variation of solutions. Partial differential equations of the second order.[c1956] tr. by HG Bergmann (Vol. 3). Dover Publications, 1964.

[2] Cannon, J.R., Esteva, S.P. and Van Der Hoek, J. A Galerkin procedure for the diffusion equation subject to the specification of mass. SIAM Journal on Numerical Analysis, 24(3), 499-515 (1987).

[3] Samarskii, A. A. Some problems of the theory of differential equations. Differentsial'nye Uravneniya, 16(11), 1925-1935 (1980). 
[4] Pandey, P.K. A Finite Difference Method for Numerical Solution of Goursat Problem of Partial Differential Equation. Open Access Libr J, 1, 1-6 (2014).

[5] Day, J.T. A Runge-Kutta Method for the Numerical Solution of the Problem in Hyperbolic Partial Differential Equations. Comput. J., 9(1), 81-83 (1966).

[6] Usman, M., Zubair, T., Ali, U. and Mohyud-Din, S.T. On Goursat Problems. Int J Mod Math Sci, 3(3), 63-76 (2012).

[7] Wazwaz, A.M. On the numerical solution of the Goursat problem. Applied mathematics and computation, 59(1), 89-95 (1993).

[8] Naseem, T. and Tahir, M. A novel technique for solving Cauchy problem for the third-order linear dispersive partial differential equation. International Journal of Physical Sciences, 8(6), 210-214 (2013).

[9] Keskin, Y., Çağlar, İ. and Koç, A. Numerical solution of sine-Gordon equation by reduced differential transform method. In Proceedings of the World Congress on Engineering. 1, 6-8 (2011).

[10] Naseem, T. and Tahir, M. Vectorial reduced differential transform (VRDT) method for the solution of inhomogeneous Cauchy-Riemann system. International Journal of Physical Sciences, 9(2), 2025 (2014).

[11] Zhou, J.K. Differential Transformation and Its Applications for Electronic Circuits, Huazhong Science \& Technology University Press, China, 1986.

[12] Adomian, G. A review of the decomposition method in applied mathematics. Journal of mathematical analysis and applications, 135(2), 501-544 (1988).

[13] Adomian, G. Nonlinear stochastic systems theory and applications to physics (Vol. 46). Springer Science \& Business Media, 1988.

[14] Adomian, G. Solving frontier problems of physics: the decomposition, With a preface by Yves Cherruault. Fundamental Theories of Physics, Kluwer Academic Publishers Group, Dordrecht, 1, 1994.

[15] Wazwaz, A.M. A reliable modification of Adomian decomposition method. Applied mathematics and computation, 102(1), 77-86 (1999).

[16] Evans, D.J. and Raslan, K.R. The Adomian decomposition method for solving delay differential equation. International Journal of Computer Mathematics, 82(1), 49-54 (2005).

[17] Hosseini, M.M. and Nasabzadeh, H. On the convergence of Adomian decomposition method. Applied mathematics and computation, 182(1), 536-543 (2006). 
[18] Chen, W. and Lu, Z. An algorithm for Adomian decomposition method. Applied Mathematics and Computation, 159(1), 221-235 (2004).

[19] Keskin, Y. and Oturanc, G. Reduced differential transform method for partial differential equations. International Journal of Nonlinear Sciences and Numerical Simulation, 10(6), 741-750 (2009).

[20] Wazwaz, A.M. and El-Sayed, S.M. A new modification of the Adomian decomposition method for linear and nonlinear operators. Applied Mathematics and computation, 122(3), 393- 405 (2001). 